\title{
Academic and Industrial Software Testing Conferences: Survey and Synergies
}

\author{
Árpád Beszédes \\ Department of Software Engineering \\ University of Szeged, Hungary \\ beszedes@inf.u-szeged.hu
}

\author{
László Vidács \\ MTA-SZTE Research Group on Artificial Intelligence \\ University of Szeged, Hungary \\ laceinf.u-szeged.hu
}

\begin{abstract}
Just as with any other profession, an efficient way to exchange ideas and networking in software testing are conferences, workshops and similar events. This is true for both professional testers and researchers working in the testing area. However, these two groups usually look for different kinds of events: a tester likes to attend "industrial" (sometimes called practitioner's or user) conferences, whereas a researcher is more likely interested in "academic" (in other words scientific or research) conferences. Although there are notable exceptions, this separation is substantial, which hinders a successful academyindustry collaboration, and communication about the demand and supply of research in software testing. This paper reviews 101 conferences: two thirds are academic ones, the rest being industrial. Besides providing this reasonably comprehensive list, we analyze any visible synergies such as events that have a mixed Program Committee and offer a program with elements from both sides. We found only a handful of such events, but these can serve both as opportunities for attendees who wish to extend their perspectives and as models for organizers of future conferences.

Index Terms-Software testing conferences, academia-industry collaboration, scientometrics.
\end{abstract}

\section{INTRODUCTION}

Successful collaboration between industry and academia in software engineering is challenging. Countless internet blogs and discussions deal with questions like "do academics teach and research what the industry requires," or "is industry aware of the latest research achievements," and - even more importantly - "do we do our best in making the two parties talk to each other?" There are also papers published in this respect, which report on possible collaboration types [1], research models [2], education and training aspects [3], for example.

Software testing is not different, and the gap between industry and academia is often investigated in this field as well [4], [5], [6], [7]. There were cases in the authors' personal experience too, where an industrial testing community was reluctant to hear about latest researches due to skepticism in the applicability of results, and conversely, research community refused to hear about an industrial case study due to the lack of significant new scientific value.

However, the exchange of research results and industry needs may be very beneficial for both academic and industrial software testing communities, and fortunately, there are a lot of initiatives for reducing this gap. The European Software Testing Innovation Alliance (EuroSTIA) [8] and the academic relations of the International Software Testing Qualifications
Board [9] are just two examples. Communication between the communities is possible via different channels, but probably the most direct and successful way are various events which give the possibility of face-to-face gathering of people to exchange ideas and networking. Such events take form of various types of conferences, workshops, symposia, etc. An interesting observation one can make is that the style, content, organization board and the intended audience of testing events also shows this typical separation into "academic" and "industrial." To foster collaboration, one way is to concentrate on possible synergies between these two types of events, encourage joint conference tracks, and attract members of both communities.

The TAIC PART - Testing: Academic and Industrial Conference on Practice and Research Techniques conference series is a good example of such an initiative. The program of almost every edition includes at least one keynote or a panel discussion about the issues of the collaboration, where the presenters identify concrete success stories and difficulties as well. There are further conferences as well, which can serve as role models to other conference organizers, and hopefully from a little more dedication to enhancing academia-industry collaboration, both communities would benefit.

The goal of the present paper is to give a systematic overview and analysis of the most visible academic and industrial software testing conferences, primarily from the synergistic point of view. It will hopefully serve as a list of offerings from both communities, with an assessment of which events provide the most of both worlds, and which are purely academic or industrial events. The internet contains several lists of academic and industrial conferences (typically only one of the two, e.g. [10] and [11]), but a systematic analysis and side-by-side presentation is not available yet. We identified 63 academic and 38 industrial conferences to include in our assessment, giving a massive list of over one hundred events. We tried to be comprehensive and identify the most visible events, and for that we worked from publicly available information. Besides providing the list, our most important finding is that there are very few events that strategically foster the synergy by including a distinct track for the other community, for instance. At the same time, the composition of the Program Committees or the invited keynotes of several conferences reflect a bit more balance in this respect. 
There are relatively few works in software engineering literature that addressed the question of conference properties. The ones that did, however, concentrate only on scientific events, such as the analysis by Vasilescu et al. [12]. To our knowledge our work is the first one to compare academic and industrial conferences.

In the next section, we give our objectives and the research method, while Sections III and IV give the list of academic and industrial conferences, respectively. The analysis of synergies is done in Section V, and we conclude in Section VI.

\section{OBJECTIVES AND METHOD}

\section{A. Objectives}

The objectives of this study can be summarized as follows:

1) Identify a reasonably comprehensive list of academic and industrial software testing conferences based on publicly available information on the internet.

2) Analyze the conferences based on the following criteria:

- general information such as the number of editions and paper/presentation type,

- composition of the organizing (program) committee,

- content, such as keynote topics and presenters, and

- any synergistic tracks, sessions or other components.

3) Classify and compare the conferences based on where they can be placed on a purely academic-joint-purely industrial scale. Some conferences have an explicit goal to promote collaboration, while others may have a dedicated track only, or no synergies at all.

4) Identify deficiencies and possibilities for enhancing future conference editions, with a set of advices for researchers, practitioners, and conference organizers.

\section{B. Research Method}

Our goal with the study was not to perform a systematic and complete review of the field (as might be suggested by some methodologies [13]). Instead, we wanted to provide a list of conferences that is reasonably complete, i.e. we used a relatively thorough search method that would a potential conference attendee use too, and if our search did not find a specific conference, it would probably be hard to find by interested attendees as well.

We searched the internet for various keywords trivially related to "software testing conference," in combination with keywords for the classification as "academic" or "industrial"/"user." The search was extended by processing any general conference listing websites we found, and other sources like blogs and discussion forums. Also, more general software engineering conferences, and related topics were included such as "software quality" and "reliability." Big conference supporters such as ACM and IEEE for academic conferences and ISTQB for industrial ones were specifically investigated for lists they publish.

For inclusion in the final list, we considered different event types including conferences and workshops. Other events like summer schools, tutorials or seminars have not been included. We tried to identify conference series where the same conference is repeated at different venues, perhaps co-located with other events, however there were cases where the event was not continued, it was renamed or merged with other events. In these cases, we relied on the data of the latest edition. Many general software engineering conferences (such as the International Conference on Software Engineering - ICSE) include tracks dedicated to various aspects of testing. We did not include these conferences; this report concentrates on dedicated events. However, co-located events like workshops are included separately, with the host conference noted. Most of the conferences we identified are international, and the information is available in English, with several exceptions which are clearly noted.

In the second phase, we analyzed the conferences for the data mentioned above using publicly available information (i.e., we did not contact conference organizers for internal data). We concentrated on the composition of the organizing team, especially Program Committee and Program Chairs. The affiliations of the members provide important information about the academic or industrial nature of the conference. Clear classification was possible in most of the cases: industrial affiliation was given if the employer was a company, and academic if it was a university or other higher education institute. We used academic affiliation as well if the organization was a publicly funded research institute.

When available, the typical content (such as the program for the latest event) has been investigated to find any synergistic tracks, keynote talks, or any other elements to support the academic or industrial nature.

Finally, the data obtained has been aggregated to get an overall rating if a conference is more of an academic or industrial nature or mixed with strong synergies. For this, we calculated an aggregated index value for each conference, which is essentially a weighted sum of the main factors influencing the synergy. Based on this information we then draw our conclusions and provide advices both for attendees and organizers of academic and industrial conferences.

\section{ACADEMIC CONFERENCES}

We list the academic conferences in alphabetical order. Several big conferences have a number of associated events, typically workshops, which are separately listed also in alphabetical order after the host conference. A unique acronym has been used to identify a conference, which was generally the official one. For each conference, we list the number of editions so far, where this information was available. Generally, the program of academic conferences is based on peer reviewed papers, which are published in the conference proceedings. The publisher of the proceedings (which is typically the main sponsor as well) is noted. In most of the cases, the papers of the co-located workshops are published in the proceedings of the main conference, so this is not separately noted. Finally, the URLs for the conference series (if available) or for the latest instance is provided. 
The following list does not include testing related tracks of more general software engineering conferences. Most notable events in this category are the International Conference on Software Engineering (ICSE), the International Conference on Software Maintenance and Evolution (ICSME), the International Conference on Software Analysis, Evolution, and Reengineering (SANER), the European Software Engineering Conference and Symposium on the Foundations of Software Engineering (ESEC/FSE), and the International Conference on Automated Software Engineering (ASE).

AISTQ - Academia Industry Software Quality \& Testing Summit: Organized by Shamoon College of Engineering Israel with support from industry, including the Israeli Testing Certification Board. Presentation possibility mostly for PhD students; selected works are considered for publication in the Inernational Journal on Software Tools for Technology Transfer (STTT) special issue. URL: http://www.sce.ac.il/aistq16/

AST - IEEE/ACM International Workshop on Automation of Software Test: A regular satellite event of the International Conference on Software Engineering (ICSE) with 11 editions so far. The workshop is sponsored and the proceedings are published by ACM. URL: http://tech.brookes.ac.uk/AST2016/

ATAMI - Advances in Testing: Academia Meets Industry: The workshop has been organized since 2010 by the Fraunhofer Institute for Open Communication Systems (FOKUS) with a dedicated goal to foster academia-industry collaboration. Proceedings are published by Fraunhofer IRB Verlag. In German. URL: http://www. fokus.fraunhofer.de/de/fokus_events/quest/atami_2013/index.html

A-TEST - Workshop on Automated Software Testing: Colocated at the Joint Meeting of the European Software Engineering Conference (ESEC) and the ACM SIGSOFT Symposium on the Foundations of Software Engineering (FSE), proceedings published as part of ESEC/FSE proceedings. One edition so far. URL: https: //staq.dsic.upv.es/A-TEST/2015/

ATSE - Workshop on Automating Test case design, Selection and Evaluation: Co-located with International Conference on Software Engineering and Formal Methods (SEFM), 6 editions so far. Proceedings are published in Springer's Lecture Notes in Computer Science. URL: https://staq.dsic.upv.es/ATSE/2015/

ATVA - International Symposium on Automated Technology for Verification and Analysis: 11 events so far, proceedings are published in Springer's Lecture Notes in Computer Science. URL: http://atva2015.ios.ac.cn/

CAV - International Conference on Computer Aided Verification: This conference had 28 editions, proceedings are published in Springer's Lecture Notes in Computer Science. URL: http://i-cav.org/2016/

ETAPS events: The European Joint Conferences on Theory \& Practice of Software (ETAPS) regularly hosts conferences related to software testing. Proceedings are published in Lecture Notes on Computer Science series by Springer. Sponsorship of ETAPS conferences is coordinated by a dedicated non-profit association. Notable conferences include:

MBT - Workshop on Model-Based Testing: Organized since 2004, mostly annual. URL: http://mbt-workshop.org/

TACAS - Tools and Algorithms for the Construction and Analysis of Systems: 22 editions so far. URL: http://www.etaps. org/2016/tacas/

ETOOS - ECOOP Workshop on Testing Object-Oriented Systems: Organized as part of the European Conference on ObjectOriented Programming (ECOOP), one edition, local proceedings. URL: http://etoos2010.sed.hu/

FM satellite events: The International Symposium on Formal Methods (FM) hosts the following testing related workshops with local proceedings:
USE - Workshop on Usages of Symbolic Execution: One edition so far. URL: http://perso.ecp.fr/ gallp/Workshop_USE.html

WWV - International Workshop on Automated Specification and Verification of Web Systems: 11 editions. URL: http://wwv2015.isti.cnr.it/

FMICS-AVoCS - International Workshop on Formal Methods for Industrial Critical Systems and Automated Verification of Critical Systems: A joint conference, with 21 editions for FMICS and 16 for AVoCS. Proceedings are published in Springer's Lecture Notes in Computer Science. URL: http://fmics-avocs.isti.cnr. it/

ICST - International Conference on Software Testing, Verification, and Validation: A premier academic software testing conference organized since 2008, which hosts several recurring workshops as well. ICST is sponsored and proceedings are published by the IEEE Computer Society. URL: http://www.cs.uic.edu/ icst2016/

ICST satellite events: Workshops of the past five ICST editions were the following:

A-MOST - Advances in Model Based Testing: 12 editions so far. URL: https://sites.google.com/site/amostw2016/

$A S Q T$ - User Symposium on Software Quality, Test and Innovation: 14 editions so far. URL: http://www.asqt.org/

CSTVA - Workshop on the Constraints in Software Testing, Verification and Analysis: 6 editions so far. URL: http: //srg.doc.ic.ac.uk/cstva14/

ESSS - International Workshop on Engineering Safety and Security Systems: Four editions. URL: http://pat.sce.ntu.edu. sg/esss15/

InSTA - International Workshop on Software Test Architecture: Three editions so far. URL: http://aster.or.jp/workshops/ insta2016/

IWCT - International Workshop on Combinatorial Testing: 5 editions so far. URL: http://iwct2016.unibg.it/

MUTATION - International Workshop on Mutation Analysis: 11 editions so far. URL: https://sites.google.com/site/ mutation2016/

REGRESSION - International Workshop on Regression Testing: Four editions so far. URL: https://sites.google.com/site/ regression2014/

SBST - International Workshop on Search-Based Software Testing: 9 editions so far. Later co-located with ICSE. See separately in this section.

SECTEST - International Workshop on Security Testing: 6 editions so far. URL: https://sites.google.com/site/ sectestworkshop15/

TAIC PART - Testing: Academic and Industrial Conference on Practice and Research Techniques: A strategically synergistic conference, with the dedicated goal to foster collaboration between academia and industry. 11 editions so far. URL: http://www2016.taicpart.org/

TESTBEDS - International Workshop on TESTing Techniques \& Experimentation Benchmarks for Event-Driven Software: Sometimes co-located with Automated Software Engineering (ASE). 6 editions so far. URL: http://www.cs.umd.edu/users/atif/ testbeds/testbeds2013.htm

TTC - International Workshop on Testing The Cloud: Sometimes co-located with International Symposium on Software Testing and Analysis (ISSTA). Two editions. URL: http://ttc2014. ucd.ie/

VOLT - Verification and validation Of model Transformations: 4 editions so far. URL: http://www.model-based-testing. de/volt12/

ICTSS - International Conference on Testing Software and Systems: 27 editions so far, formerly known as Conference on Testing of Communicating Systems (TESTCOM) and Workshop on Formal Approaches to Testing of Software (FATES). Sposored by 
International Federation for Information Processing (IFIP), proceedings are published in Springer's Lecture Notes in Computer Science. URL: http://www.aus.edu/ictss15

iFM satellite events: The International Conference on integrated Formal Methods (iFM) conference series is held since 1999 approximately every second year, and it regularly includes various workshops related to formal aspects of testing, verification and validation. Conference proceedings are published in Springer's Lecture Notes in Computer Science. URL: http://ifm2016.ru.is/ Notable conferences include:

PrePost - Pre- and Post-Deployment Verification Techniques: One edition so far. URL: http://icetcs.ru.is/prepost/

V2CPS - Workshop on Verification and Validation of Cyber-Physical Systems: One edition so far. URL: http://www. cs.ox.ac.uk/conferences/VVCPS16/

INTUITEST - International Workshop on User Interface Test Automation: Partially sponsored by the European Telecommunications Standards Institute (ETSI). One edition so far, proceedings published by Fraunhofer IRB Verlag. URL: https://staq.dsic.upv.es/ INTUITEST/2015/

ISoLa - International Symposium on Leveraging Applications of Formal Methods, Verification and Validation: This conference is organized from 2004 mostly every year, with partial sponsorship from the European Association for the Study of Science and Technology (EASST). Proceedings are published in Springer's Lecture Notes in Computer Science. URL: http://www. isola-conference.org/

ISSRE - IEEE International Symposium on Software Reliability Engineering: A conference with many common points with software testing, organized since 1996. Very strong industrial presence. ISSRE hosts several workshops which are also related to software testing. ISSRE is sponsored and proceedings are published by IEEE Computer Society. URL: http://www.issre.net/

ISSTA - International Symposium on Software Testing and Analysis: A premier academic software testing conference sponsored by ACM Special Interest Group on Software Engineering (SIGSOFT). The first edition was held in 1993, and is organized yearly since 2006. ISSTA proceedings are published by ACM. URL: https://issta2016.cispa.saarland/

ISSTA satellite events: The conference hosts several recurring workshops as well; events of the last five editions were the following:

CHESE - International Code Hunt Workshop on Educational Software Engineering: One edition so far. URL: http: //research.microsoft.com/chese2015

ETSE - International Workshop on End-to-end Test Script Engineering: Two editions so far. URL: https://sites.google.com/ site/etse2012workshop/

FutDeb - The Future of Debugging: Two editions so far. URL: https://sites.google.com/site/futdeb2013/

JAMAICA - Workshop on Joining AcadeMiA and Industry Contributions to Test Automation and Model-based Testing: Dedicated to foster academia-industry collaboration, two editions so far. URL: http://www.model-based-testing.de/jamaica14/

PADTAD - Workshop on Parallel and Distributed Systems: Testing, Analysis, and Debugging: 10 editions. URL: http://faculty.uoit.ca/bradbury/padtad2012/

PERTEA - Workshop on Software and System Performance Testing, Debugging, and Analytics: One edition so far. URL: http://woda14.cs.rutgers.edu/

QASBA - International Workshop on Quality Assurance for Service-based Applications: Two editions so far. URL: http: //fmt.isti.cnr.it/qasba2013/

QuoMBaT - Quality of Model-Based Testing: Two editions so far. URL: http://www.model-based-testing.de/quombat13

SPIN - International SPIN Symposium on Model Checking of Software: See separately in this section.
SSEAT - Workshop on State-space Exploration for Automated Testing: Four editions so far. URL: https://sites.google.com/ site/sseatws/

WODA - International Workshop on Dynamic Analysis: Organized since 2003, sometimes co-located with other conferences. URL: http://woda15.ics.uci.edu/

LT - International Workshop on Large-Scale Testing:

A regular workshop of the ACM/SPEC International Conference on Performance Engineering (ICPE), organized yearly from 2012. Sponsored by ACM SIGMETRICS, SIGSOFT, and SPEC, proceedings published by ACM. Earlier co-located with ICST. URL: http://lt2016.eecs.yorku.ca/

MET - International Workshop on Metamorphic Testing: A first edition of the workhop at the International Conference on Software Engineering (ICSE). Workshop is sponsored and the proceedings are published by ACM. URL: http://www.cs.montana.edu/met16/

MoDeVVa - Workshop on Model Driven Engineering, Verification, and Validation: Organized yearly since 2004, this workshop is a regular satellite event of the ACM/IEEE International Conference on Model Driven Engineering Languages and Systems (MODELS). Published as CEUR Workshop Proceedings. URL: https: //sites.google.com/site/modevva/

NFM - NASA Formal Methods Symposium: The conference is organized since 8 years by NASA's Formal Methods Group in collaboration with various universities. Proceedings are published in Springer's Lecture Notes in Computer Science. URL: http://crisys.cs. umn.edu/nfm2016

PAS - International Seminar on Program Verification, Automated Debugging and Symbolic Computation: 4 events so far, local proceedings. URL: http://pas2015.cc4cm.org/

QRS - IEEE International Conference on Software Quality, Reliability \& Security: Formerly separated as IEEE International Conference on Software Security and Reliability (SERE) and IEEE International Conference on Quality Software (QSIC), this conference is sponsored and the proceedings are published by IEEE Reliability Society. URL: http://paris.utdallas.edu/qrs16

QUATIC - International Conference on Quality of Information and Communications Technology: With 10 editions so far starting from 1994, this conference is organized by various Portugiese universities and research institutes. Proceedings are published by Conference Publishing Services (CPS). URL: http://2016.quatic.org/

RET - International Workshop on Requirements Engineering and Testing: A relatively new workshop with 3 editions so far, co-located with various conferences: IEEE International Requirements Engineering Conference (RE), the International Conference on Software Engineering (ICSE), International Working Conference on Requirements Engineering: Foundation for Software Quality (REFSQ). Published as CEUR Workshop Proceedings. URL: http: //ret.cs.lth.se/16/

$R V$ - International Conference on Runtime Verification: Organized since 2001, proceedings are published in Springer's Lecture Notes in Computer Science. URL: https://rv2016.imag.fr/

SAC-SVT - Software Verification and Testing: A Track of the ACM Symposium on Applied Computing (SAC), yearly organized since 1986. SAC is sponsored by the ACM Special Interest Group on Applied Computing (SIGAPP), proceedings are published by ACM. URL: http://antares.sip.ucm.es/svt16/

SAST - Brazilian Workshop on Systematic and Automated Software Testing: This workshop is organized since 2007 by various Brazilian universities, with locally published proceedings. URL: http://sast2015.icmc.usp.br/

SBST - International Workshop on Search-Based Software Testing: From 2008-2013, the workshop was co-located with International Conference on Software Testing, Verification and Validation (ICST), since 2014 it has been co-located with International Conference on Software Engineering (ICSE), and the papers are published as ICSE Workshop Proceedings in the ACM and IEEE Digital Libraries. URL: http://www.searchbasedsoftwaretesting.org/ 
SPIN - International SPIN Symposium on Model Checking of Software: Held in conjunction with various conferences including ETAPS, ISSTA and separate, this conference is sponsored by various organizations. 23 editions so far. Proceedings are published in Springer's Lecture Notes in Computer Science. URL: http://www.spin2016.info/

SQM - International Workshop on Software Quality and Maintainability: Co-located with the European Conference on Software Maintenance and Reengineering (CSMR), now International Conference on Software Analysis, Evolution, and Reengineering (SANER). Eight editions. Sponsored and proceedings published by IEEE with the main conference. URL: http://sqm2014.sig.eu/

STAM - IEEE International Workshop on Security Testing and Monitoring: Co-located with the IEEE International Conference on Distributed Computing Systems (ICDCS), two editions so far. Sponsored and proceedings published by IEEE with the main conference. URL: http://www.montimage.com/STAM2016/

STV - Workshop on System Testing and Validation: The workshop has been organized since 2002 almost every year by the Fraunhofer Institute for Open Communication Systems (FOKUS). It has been co-located with various international conferences. Proceedings published by Fraunhofer IRB Verlag. Large industrial presence. URL: https://www.fokus.fraunhofer.de/go/stv15

TAP - International Conference on Test And Proofs: Part of Software Technologies: Applications and Foundations (STAF) conference series organized and sponsored by various Austrian academic institutions. Proceedings are published in Springer's Lecture Notes in Computer Science. URL: http://tap2016.ist.tugraz.at/

VALID - International Conference on Advances in System Testing and Validation Lifecycle: Sponsored by the International Academy, Research, and Industry Association (IARIA), co-located with other events of the SoftNet network. URL: http://www.iaria.org/ conferences2016/VALID16.html

VST - International Workshop on Validating Software Tests: Co-located with the IEEE International Conference on Software Analysis, Evolution, and Reengineering (SANER), this is the first instance of the workshop. Papers published by IEEE with the proceedings of the main conference. URL: http://www.itri.aist.go.jp/ events/vst-2016-cfp.html

\section{INDUSTRIAL CONFERENCES}

Industrial software testing conferences differ from academic ones in several aspects. They typically do not include proceedings with papers, as the talks are not accompanied with peer reviewed papers (there are some exceptions, though). If known, we provide the format of the talk/paper submissions in the list below. Another big difference is that sponsorship is typically based on industrial support rather than with the help of a general organization like IEEE or ACM, which is the case for many academic conferences. Some industrial conferences are supported (and organized) by companies or other technical organizations. Also, industrial conferences typically do not host satellite events, rather the program is composed of various tracks, product exhibitions, tutorials, keynotes, etc.

For many industrial conferences, it is typical that they are international but organized locally, always in the same country or region. Others are highly localized, often held in the language of the given country.

The following list includes industrial conferences in alphabetical order. The International Software Testing Qualifications Board (ISTQB) [9] maintains a list of industrial conferences, which are recognized by the organization. These conferences are listed jointly, also in alphabetical order. For the identification of the events, a unique acronym was used, which was in several cases invented by the authors of this paper because the conference did not use an own acronym. We must note that it was much harder to obtain the necessary information for these conferences than for the academic ones. We will explain how we dealt with missing information in Section V.

An interesting note is that several conferences from this list are supported by the Speak Easy program, to help inexperienced speakers prepare for their talk [14].

As in the case of academic conferences, we provide the URLs for the conference series (if available) or for the latest instance, and the number of instances so far, if known.

AADAYS - Agile \& Automation Days: Mostly local conference organized in Poland with industrial support. Talks based on invitation. URL: http://aadays.pl/

ATD - Agile Testing Days: A European annual conference organized since 2012 by Díaz \& Hilterscheid $\mathrm{GmbH}$ with industrial support. Mostly exhibitions, tutorials and product presentations. There are local editions as well such as the Agile Testing Day Netherlands. URL: http://www.agiletestingdays.com/

ATD - Australian Testing Days: Organized by the Test Engineering Alliance Melbourne (TEAM) with industry support, first edition. Associated satellite events. Presentations based on open call. URL: http://testengineeringalliance.com/ australian-testing-days-2016/

BTD - Belgium Testing Days: Mostly local conference but with international presence, organized since 2009. URL: http://btdconf. com/

CAST - Conference of the Association for Software Testing: Organized yearly by the Association for Software Testing (AST) since 2006. Possibility for a full paper published in the conference proceedings. URL: http://www.associationforsoftwaretesting.org/conference/ cast-2016/

CC - Copenhagen Context: Three editions so far, organized and supported by various Danish companies. Presentations based on open call. URL: http://copenhagencontext.com/

DTC - Dutch Testing Conference: Organized since 2009, local conference, most information is in Dutch. URL: http:// dutchtestingconference.nl/

DTD - Dutch Testing Day: 21 editions so far. Industrial sponsorship, presentation based on invitation and open call. URL: http://www.testdag.nl/index.php/en/

ETC - European Testing Conference: Industrially sponsored conference, including the Association for Software Testing (AST). Presentations are selected based on invitation and open call. URL: http://europeantestingconference.eu/

EuroSTAR - EuroSTAR Software Testing Conference: Organized yearly since 1993 in various European cities. Sponsored by different supporting organizations including the Specialist Group in Software Testing (SIGIST) of the British Computer Society (BCS). Presentations are selected based on invitation and open calls. URL: http://www.eurostarconferences.com/

GTD - German Testing Day: Organized since 2011, local conference, most information is in German. URL: http://www. germantestingday.info/

ICSQ - ASQ International Conference on Software Quality: Organized by the Software Division of the American Society for Quality (ASQ) with industrial sponsorship. Talks based on invitation. URL: http://www.asq-icsq.org/

ISTQB Conference Network conferences: The ISTQB supports industrial software testing conferences that are organized or recognized by one of its member boards or partners, as listed below. URL: http://www.istqb.org/special-initiatives/ istqb-conference-network.html 
ANZTB Test - Advancing Testing Expertise Conference: Mostly local conference organized by the Australia and New Zealand Testing Board with industrial sponsorship. Talks based on invitation. URL: http://anztb.org/events.php

$A S T Q B$ - Software Testing Conference: International conference organized by the American Software Testing Qualifications Board with industrial sponsorship. Talks based on invitation. URL: http://www.astqb.org/certified-tester-resources/ astqb-software-testing-conference/

CSTQB - International Software Testing Forum: International conference organized by the Chinese Software Testing Qualifications Board with industrial sponsorship, with 6 editions so far. No formal papers and proceedings, but submission of talk proposals possible. URL: http://www.cstqbforum.com/en/

DSTB - Softwaretest: Mostly local conference organized by the Danish Software Testing Board with industrial sponsorship. No formal papers and proceedings, but submission of talk proposals possible. URL: http://dstb.dk/

DSTC - STeP-IN Forum Conference: Continuation of STePIN Summit conference series (13 editions so far), organized by STePIN Forum India with industrial sponsorship. No formal papers and proceedings, but submission of talk proposals possible. URL: http: //dstc.stepinforum.org/

HUSTEF - Hungarian Software Testing Forum: International conference organized by the Hungarian Testing Board with industrial sponsorship, 5 editions so far. No formal papers and proceedings, but submission of talk proposals possible. URL: http: //www.hustef.hu/

IQNITE - Conference for Software Quality and Testing: A German conference organized and sponsored by the Software Quality Systems Group (SQS), with 19 editions so far. Also organized in other countries such as United Kingdom and Australia. Presentations based on invitation. URL: http://www.iqnite-conferences.com/

NTD - Nordic Testing Days: Annual conference organized by the Estonian Testing Board with industrial support. No formal papers and proceedings, but submission of talk proposals possible. URL: http://nordictestingdays.eu/

SEETEST - South East European Software Testing Conference: International conference organized by the South East European Testing Board with industrial sponsorship with 4 editions so far. Talks based on invitation. URL: http://seetest.org/

STF - Italian Software Testing Forum: Mostly local conference organized by the Italian Software Testing Qualifications Board with industrial sponsorship. Talks based on invitation. URL: http://www.swtestingforum.org/

SWQD - Software Quality Days Conference: Software Quality Days is organized since 2009. It hosts various types of presentations, lectures, and papers, including a scientific track. Presentations are selected based on invitation and open calls. Funding is based on industrial sponsorship. Separate Program Committee for the scientific track. Papers from the scientific track are published by Springer, the others in a local conference publication. URL: http://www.software-quality-days.com/

PNSQC - Pacific NW Software Quality Conferences: Organized since 2006, sponsored by industrial partners. Possibilities for presentations, posters, or papers. URL: http://www.pnsqc.org/

LETS - Let's Test Conference: Organized since 2012 with industrial sponsorship. Talks based on invitation, a mostly informal event. URL: http://lets-test.com/

PSQT - Practical Software Quality and Testing: Organized by the International Institute for Software Testing with industrial sponsorship. No formal papers and proceedings, but submission of talk proposals possible. URL: http://www.psqtconference.com/

$Q A-Q A$ or the Highway: Regional conference organized in Columbus, Ohio, USA, three editions so far. No formal papers and proceedings, but submission of talk proposals possible. URL: http: //qaorthehighway.com/
QATEST - International Conference on Software Quality Assurance and Testing on Embedded Systems: Organized and sponsored by the Software Quality Systems Group (SQS) since 2001. No formal papers and proceedings, but submission of talk proposals possible. URL: http://www.qatest.org/

$Q S$ - Software QS-Tag: Mostly German conference organized and sponsored by Imbus AG since more than 20 years. Mostly exhibitions and product presentations. URL: http://www.qs-tag.de/

QUEST - Quality Engineered Software \& Testing Conference \& Expo: Organized by Quality Assurance Institute (QAI) Global since 2008. No formal publications, presentation based on invitation and sponsorship. URL: http://www.qaiquest.org/

RTC - Romanian Testing Conference: Organized yearly since 2012 by an informal testing community with industrial support. No formal papers and proceedings, but submission of talk proposals possible. URL: http://www.romaniatesting.ro/

SIGIST Conference: Conference series organized by the Specialist Group in Software Testing (SIGIST) of the British Computer Society (BCS) since 2008 four times a year. Talks based on invitation. There are international events organized by SIGIST as well, for instance SIGIST Israel Conference. URL: http://www.bcs.org/category/ 9264

STC - Annual International Software Testing Conference: Organized by Quality Assurance Institute (QAI) with 15 editions so far, mostly in India. Possibility for formal paper submission. URL: http://qaistc.com/

STPCon - Software Test Professionals Conference \& Expo: Organized by the Software Test Professionals (STP) association, industrial sponsorship. Presentations based on invitation only. URL: http://www.stpcon.com/

TAD - Test Automation Day: Organized by CKC Seminars in the Netherlands, 5 editions so far. No formal papers and proceedings, but submission of talk proposals possible. URL: http: //www.testautomationday.com/

TechWell conferences: TechWell Corporation organizes and sponsors a series of software testing conferences in various parts of North America: Software Testing Analysis \& Review - East Conference (STAR EAST), Software Testing Analysis \& Review West Conference (STAR WEST), STAR CANADA, Better Software Conference West, Better Software Conference East, Mobile Dev + Test Conferences, IoT Dev + Test Conferences. No formal paper or proceedings. According to the website, these conferences are "the longest-running software testing events in the industry." URL: https://www.techwell.com/software-conferences/

TestBash - Brighton: A conference organized by the Software Testing Club Ltd. with industrial support. Talks based on invitation, mostly informal event. URL: http://www.ministryoftesting.com/ training-events/testbash-brighton-2016/

UCAAT - User Conference on Advanced Automated Testing: Organized and partially sponsored by the European Telecommunications Standards Institute (ETSI), with additional sponsorship from industry. Annual event since 2013. Formerly Model-Based Testing User Conference (MBTUC). Presentation based on invitation and open call, no formal papers and proceedings. URL: http://ucaat.etsi. org/2015/

\section{ANALYSIS OF SYNERGIES}

The two types of conferences listed in the previous sections differ in many aspects. Most notably, academic conferences are based on presentations accompanied by peer reviewed papers, while industrial ones often involve more informal submissions but a big focus is put on the keynotes. Generally, academic conferences were typically much easier to analyze for any synergies because the organization is more transparent: the key 
organization roles and detailed programs were usually available, which was not the case with the industrial conferences.

In the following, we first separately analyze the two categories, and then in Section $\mathrm{V}-\mathrm{C}$, we summarize our findings. There are general properties such as committee sizes and age, which can directly be compared. But for the comparison of the synergistic aspects, we will use an aggregated measure for each conference, which will have two variants, one for the academic and one for industrial conferences.

For the analysis of synergies, we extracted the following properties for each conference:

- Age of the conference in number of editions so far.

- Number and affiliations (academic or industrial) of Program Chairs.

- Size and composition (academic or industrial) of the Program Committee.

- Number and affiliations (academic or industrial) of Keynote speakers.

- A synergistic factor which we determined based on our subjective judgement: we considered if the conference explicitly sets an objective to provide a synergy in form of, for instance, a dedicated industrial or academic track, respectively.

In each case, the information about the latest event has been used, which was in many cases the upcoming event for 2016.

As expected, there were cases when the information was missing or unreliable. In these cases we defaulted to $100 \%$ academic or industrial composition for the two categories, respectively. In the case of academic conferences, only for ATAMI we could not find details neither about the Program Chairs nor the Program Committee. In four other cases, the composition of the Program Committee was unknown. In 13 cases, the Keynotes were not available. The situation was much worse in the case of industrial conferences. Except for five events, either Program Chair or Program Committee information was missing. The chairs were usually known, but in 13 cases out of 38 , the committee information was missing. This could perhaps mean that there was no formal committee and the chairs did the selection. However, information about the Keynote was missing only in four industrial conferences.

Apart from the keynote speakers and the synergistic track, we did not analyze the programs of the conferences, although it might be an interesting additional aspect. First, this would require the analysis of more previous editions, and to make a common classification of topics. Second, the industrial/academic synergy is typically not visible from the topic itself, rather it depends on each individual paper. Considering this dimension is left for future work.

\section{A. Academic Conferences}

We found altogether 63 events in this category including large conferences and smaller satellite events and workshops.

1) General properties: The structure of most academic conferences is similar: they expect submissions based on calls for papers. The submissions are peer reviewed and the accepted papers are published in conference proceedings. The proceedings are in many cases published by a high profile institution such as IEEE, ACM or Springer. Some of the workshops publish their proceedings locally or at a less significant publisher. Although we do not have data about attendance, the format of the conferences is most suitable for researchers, so we expect that the majority of attendees come from academia.

The average age of academic conferences is 8.1 years, the longest running events are 25-28 years old, while 11 conferences had only one edition so far.

Statistics about the organization team and keynotes for academic conferences is given in Table I. For each role, the number of persons with industrial and academic affiliations are given in columns 2 and 3 as a sum over all investigated conferences (their last editions). The fifth column shows the ratio of industrial persons in percents. The average sizes of the groups show no surprises, except the Program Chair category. Most of the conferences have one or two chairs in these roles, however for several workshops there were no separate persons named in these roles. In these cases we used the number of the organizers of the workshops, which raises the average number.

TABLE I

ACADEMIC CONFERENCES: PERSONS INVOLVED IN MAIN ROLES

\begin{tabular}{|l|c|c|c|c|c|}
\hline Role & I & A & Sum & I \% & Avg. size \\
\hline PC/OC chair & 26 & 159 & 185 & $14 \%$ & 2.9 \\
PC member & 220 & 1299 & 1519 & $14 \%$ & 24.1 \\
Keynote & 30 & 76 & 106 & $28 \%$ & 1.7 \\
\hline All roles & 276 & 1534 & 1810 & $15 \%$ & - \\
\hline
\end{tabular}

2) Industrial synergies: The first indicator of industrial synergies in academic conferences are the ratios of members with industrial affiliations in each of the roles from the previous table. This information can also be seen graphically in Figure 1 (left-hand side bars). The program chairs and committee has a modest industrial representation of slightly above $10 \%$, but the ratio of industrial keynotes is more significant. About one quarter of keynotes given at all of the investigated conferences came from industry, which is very plausible. We believe that organizers of academic conferences try to make their events more attractive (both for researchers and practitioners) by inviting industrial people, however the composition of the program could also benefit from a bit more industrial presence.

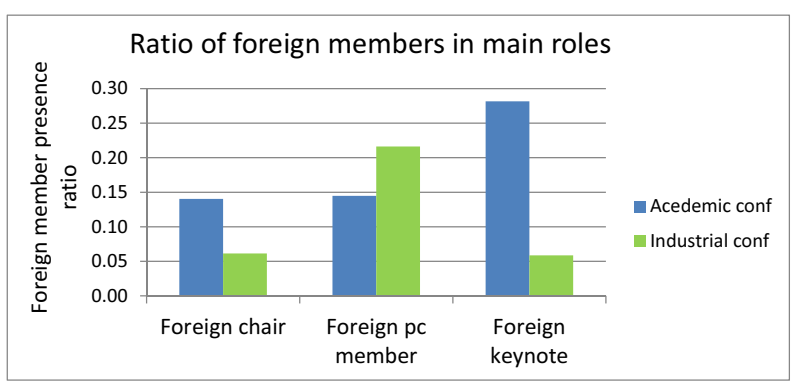

Fig. 1. Presence ratio of industrial members in main conference roles in academic conferences and vice versa 
To be able to express in a single comparable value the level of industrial synergies for each conference, we apply a simple model that involves the mentioned basic statistics and an additional synergistic factor. We define the Industry Index as the following weighted average:

$$
I I=\frac{w_{c h} I R_{c h}+w_{p c} I R_{p c}+w_{k e y} I R_{k e y}}{w_{c h}+w_{p c}+w_{k e y}} \cdot(I S+1),
$$

where $w_{x}$ are the weights for program chairs, program committee and keynote speakers, respectively. We subjectively determined the following weight factors: $w_{c h}=3, w_{p c}=2$ and $w_{k e y}=1$ with the rationale that the selection of the program primarily depends on the program chairs, then on the program committee, while the affiliation of the keynotes many times serves only "marketing" purposes and adds little to the actual program of the conference. $I R_{x}$ are the industryratios for each category, which are calculated as the ratio of the number of persons with industrial affiliations over the number of all persons in the given category.

We also use the additional Industrial Synergy factor IS, which is basically our subjective categorization for each conference, which might increase the weighted average if we determine that other factors contribute to the synergy apart from the statistics. We used two primary aspects for determining this factor. First, if the conference description explicitly states that they have a strategic goal to foster academy-industry collaboration. Second, we looked at any evidence supporting this such as if there are specific tracks or submission types for industrial results. This factor can take values in the interval $0-1$, where 0 means no industrial objectives, and 1 means that the conference has as an explicit goal to promote academyindustry collaboration. In the analysis we used 0.5 as well for conferences which are basically academic in nature but have at least a separate industrial track, industrial committee or other similar component. Conferences with $I S$ value of 1 were AISTQ, ATAMI, ISSRE, JAMAICA, STV and TAICPART. We gave 0.5 score to the following events: ASQT, ICST, LT, QASBA, RET, STAM and TESTBEDS.

The II values for the academic conferences range in a wide scale. The minimum and maximum values were 0 and 1.19 , respectively. The average was 0.17 , and the median 0.08 . Based on this index, the top-7 (upper 10\%) mostly industrysynergistic conferences are: JAMAICA (1.19), AISTQ (0.79), ASQT (0.54), InSTA (0.50), A-TEST (0.48), STV (0.42), and TAIC-PART (0.41). 33 conferences have an index below 0.10 .

We compared the index values with the age of the conferences and discovered an interesting trend. As Figure 2 shows, younger conferences tend to focus more on industry. This chart shows the average index values separated for conferences that had more than three instances and younger ones. There is a notable difference between young and mature conferences, which can also be observed in Figure 3. It shows the same data on a longer time scale (the data are accumulated, meaning that the average was computed for conferences of a given age or older, and we excluded events that had only one instance), indicating a positive trend.

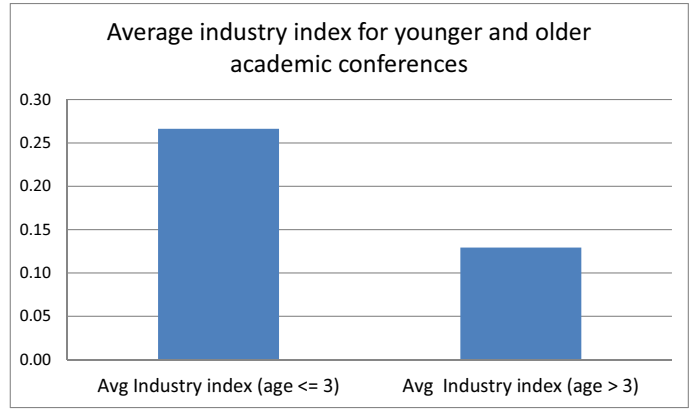

Fig. 2. Average industry index of young and mature conferences

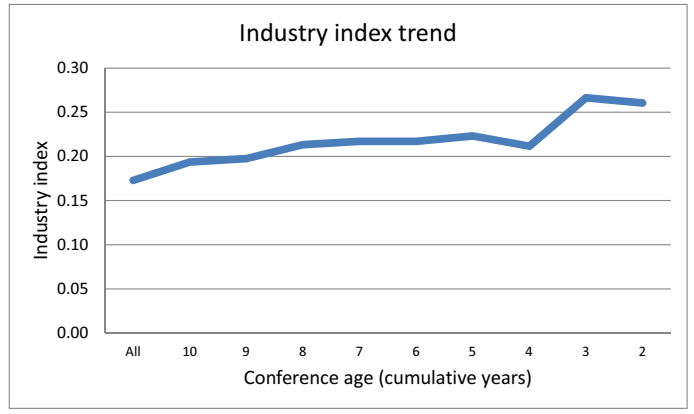

Fig. 3. Average industry index trends in academic conferences

\section{B. Industrial Conferences}

We investigated 38 industrial conferences and, as mentioned, there were more missing information here than for the academic events. This mostly concerns the organizing team. To deal with this issue we either did not count the conference with missing information (such as for an average), or we defaulted to purely industrial (such as for committee composition ratio).

1) General properties: As opposed to academic conferences, industrial ones typically do not offer the possibility for publishing a peer-reviewed paper. Instead, presentations are either based on direct invitation or by selecting from submissions to a call for presentations. Such submissions are evaluated by a program committee or, as is often the case, the selection is done directly by the organizing team (program chairs). If there is a proceedings, it is typically published locally. Unfortunately, we found out that submitting a formally peer-reviewed paper which is published in a proceedings is possible at only four of the industrial conferences (CAST, PNSQC, SWQD and STC). Similarly to academic conferences, we were not able to get data about the attendees of industrial conferences. But it is also to be expected that the majority of the audience are practitioners with industrial affiliations.

The average age of industrial conferences was 6.6 years, which is a bit less than for the academic ones (the information about the editions so far was missing in 13 cases).

Table II shows statistics about the organization teams and keynote speakers of industrial conferences. The structure of the table is similar to that of Table I with the difference that here we highlight the ratio of academic members in the committees in column 5, and not industrial ones. Compared to academic 
conferences, the average number of chairs is similar, but the program committee is much smaller. This probably reflects the simpler task of selecting the applications. Another notable difference is the average number of keynotes. This number is high because in several cases we could not distinguish between a regular talk and a keynote talk at a conference, they were are listed on the conference websites simply as "presentations." In these cases we counted all such talks.

TABLE II

INDUSTRIAL CONFERENCES: PERSONS INVOLVED IN MAIN ROLES

\begin{tabular}{|l|c|c|c|c|c|}
\hline Role & I & A & Sum & A \% & Avg. size \\
\hline PC/OC chair & 46 & 3 & 48 & $6 \%$ & 1.3 \\
PC member & 210 & 58 & 268 & $22 \%$ & 7.3 \\
Keynote & 179 & 11 & 187 & $6 \%$ & 5.1 \\
\hline All roles & 435 & 72 & 503 & $14 \%$ & - \\
\hline
\end{tabular}

2) Academic synergies: For the analysis of possible synergies in industrial conferences we used a similar approach to the academic ones. Figure 1 shows the ratios of academic members from the previous table in the right-hand bars. There are several notable differences to the academic conferences. The ratio of the chairs is a bit worse, meaning that industrial conferences like to keep the decision in the hands of industrial people. However, the number of academic members in program committees is higher than in the opposite case. This is probably because many industrial conferences like to promote their competence by inviting academic people. We must also note here that one of the conferences, SWQD, has a separate academic program committee which raises this number significantly. This conference has 4 academic and 11 industrial members in the regular track, and an additional 33 members from academia for its research track. Finally, it is interesting to observe that industrial conferences invite keynote speakers from academia on a much rarer occasion, which is not a good approach to foster collaboration. To express the aggregated level of academic synergies, we used a similar model to what we used in the academic case. The Academia Index is calculated as the following weighted average:

$$
A I=\frac{w_{c h} A R_{c h}+w_{p c} A R_{p c}+w_{k e y} A R_{k e y}}{w_{c h}+w_{p c}+w_{k e y}} \cdot(A S+1),
$$

where the notation is similar as for $I I$, except that $A R$ means academic ratio, that is the ratio of people with academic affiliations in the roles, and $A S$ means our categorization of academic synergy on a similar scale to $I S$. In this case, we found very few conferences that fit to our criteria: namely, only SIGIST and SWQD got a score of 1, while STC and UCAAT got 0.5. Among these, we emphasize SWQD, which is unique in that it includes a separate scientific track with a separate program committe, peer-reviewed papers and proceedings.

The overall distribution of the $A I$ values was different than for $I I$. The minimum value was 0 and the maximal 1.01 . But the average was only 0.06 , and the median was 0 (because as much as 25 conferences out of 38 have a zero index). Highest ranking conferences having at least 0.10 index (these are 8 conferences which constitute the upper $21 \%$ ) are: SWQD
(1.01), CAST (0.25), UCAAT (0.17), TAD (0.15), CSTQB (0.14), HUSTEF (0.13), DTD (0.13) and IQNITE (0.10).

In the case of industrial conferences we did not notice any interesting trends with respect to the age of the conference and the academic index.

\section{Summary and possibilities for improvement}

We may summarize our most notable findings from the previous sections as follows:

- Academic conferences more often invite industrial members as program chairs than industrial conferences invite academic ones.

- Synergies regarding the membership of program committees are similar.

- There are more keynotes with industrial background on academic conferences than academic keynotes on industrial ones.

- Considering other factors as well, most notably the existence of a synergistic track, academic conferences show more ambitions to synergies, which is reflected in our overall academic- and industrial indices.

- In the case of academic events, it seems that younger conferences are more industry oriented than the more mature ones.

We identified only a handful of conferences that perform exceptionally well in terms of synergies. The top rated academic conferences listed in Section V-A and the top industrial ones from Section V-B can serve as role models for organizers of future conferences. An overall picture of the landscape of conferences is shown in Figure 4. Here, we show conferences of both types in a graphical from in which each dot corresponds to a particular event. The color and the shape determines its type, while the size of the symbol is proportional to the respective academic or industrial index. The placement of the dots in the two-dimensional space reflects other two important attributes: the $\mathrm{x}$ axis shows the age of the conference in years, while we placed dots along the $y$ axis based on the size of the Program Committee. We treat the latter attribute as a proxy to the conference size (and perhaps its significance). The graph has been trimmed in both directions to exclude the 2 outliers and give better visibility to the majority.

\section{CONCLUSIONS}

In this paper, we listed what we believe to be a reasonably comprehensive set of academic and industrial software testing conferences. Our more in depth analysis of possible synergies revealed some interesting insights, which we believe will be useful for researchers and practitioners attending these events, but also for organizers of future conferences from both sides.

Overall, we are not really satisfied with the level of synergies these conferences demonstrate. There seems to exist a resistance at both sides for more extensive opening towards the other community. There are exceptions, though: conferences like TAIC-PART or SWQD are great venues to foster academic-industrial collaboration in software testing, and are examples of mutual recognition between the two communities. 


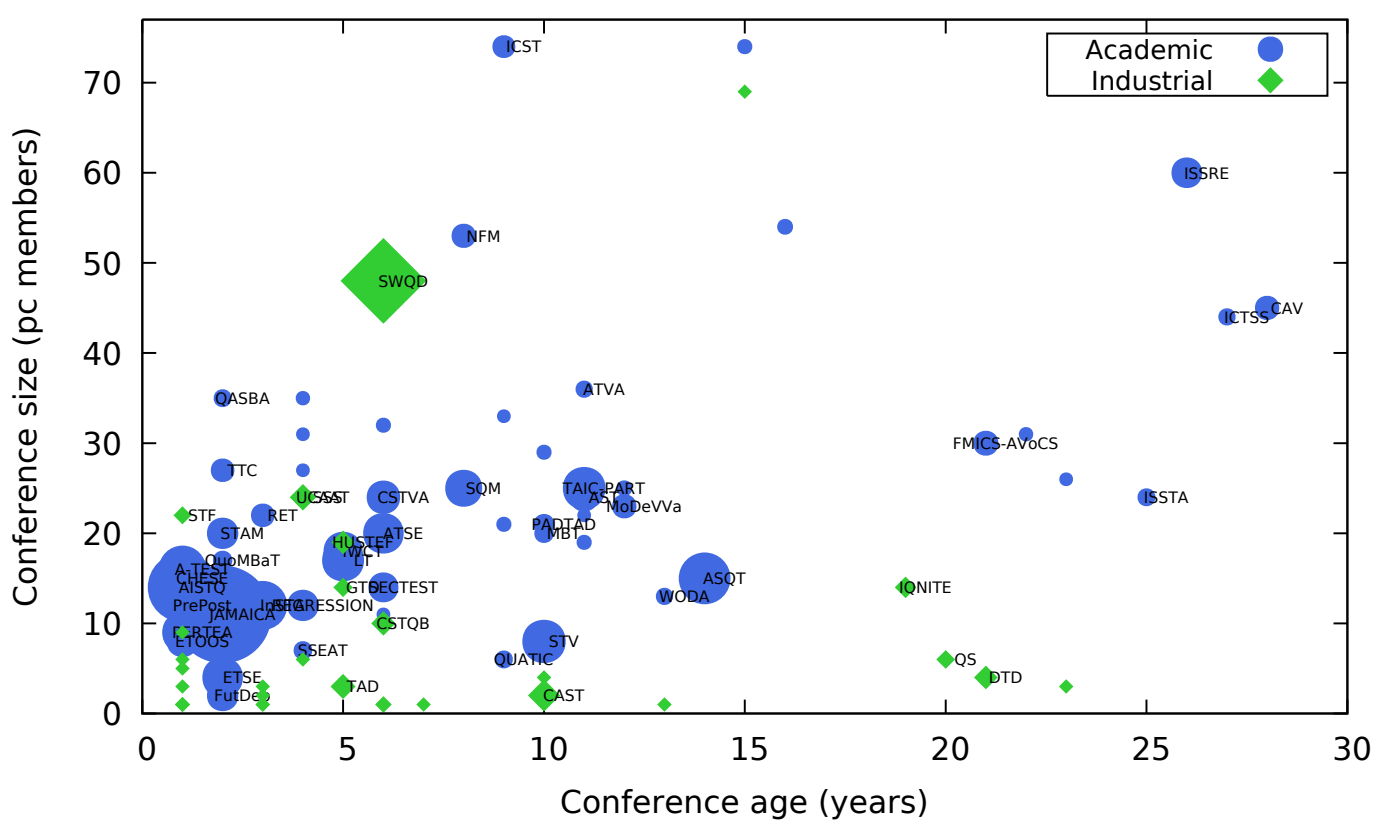

Fig. 4. Comparison of synergies (to help readability names for conferences with small indices are not shown)

Our advise for researchers who regularly attend academic conferences is that they should take a look at possible industrial events for presenting their work. Some of them even have the possibility for publishing peer-reviewed papers, which is a very important aspect for many academics. As for our advice for the practitioners, we believe that there are several highprofile academic conferences that offer a very strong technical program, and that software testing research is not only about "useless theoretical problems" but many researches address industrially driven problems and offer usable solutions.

The organizers of many great software testing conferences are trying to improve synergies, but there are lots of possibilities for improvement. In particular, we suggest adding more dedicated tracks and associated calls for contributions for both communities. A further advice for organizers of industrial conferences is that they should make sure to be more transparent regarding the organization teams and program.

We carefully performed the analysis presented in this paper, but there are a number of improvement and extension possibilities. For example, it would be possible to contact conference organizers asking for more detailed and precise information, particularily about the participants and program composition. The analysis of earlier editions of individual conferences and not only the latest ones would reveal individual trends. A future study could also consider analyzing conferences with different sizes and maturity separately. Finally, surveying conference participants and other professionals for their subjective opinions about software testing events would be a very interesting task for future research.

The raw data used for the analysis in this paper is available from the authors. In the future, we also plan to set up an online repository for the data with query possibilities.

\section{REFERENCES}

[1] P. N. Robillard and M. P. Robillard, "Types of collaborative work in software engineering," Journal of Systems and Software, vol. 53, no. 3, pp. 219-224, 2000.

[2] K. Petersen, C. Gencel, N. Asghari, D. Baca, and S. Betz, "Action research as a model for industry-academia collaboration in the software engineering context," in Proceedings of the 2014 International Workshop on Long-term Industrial Collaboration on Software Engineering, ser. WISE' 14. New York, NY, USA: ACM, 2014, pp. 55-62.

[3] Manisha and M. Manuja, "Industry academia collaboration model: The design challenges," in Software Engineering Education and Training (CSEE T), 2011 24th IEEE-CS Conference on, May 2011, pp. 111-120.

[4] R. Glass, R. Collard, A. Bertolino, J. Bach, and C. Kaner, "Software testing and industry needs," Software, IEEE, vol. 23, no. 4, pp. 55-57, July 2006.

[5] A. Bertolino, "The (im)maturity level of software testing," SIGSOFT Softw. Eng. Notes, vol. 29, no. 5, pp. 1-4, Sep. 2004.

[6] - "Software testing research and practice," in Proceedings of the Abstract State Machines 10th International Conference on Advances in Theory and Practice, ser. ASM'03. Berlin, Heidelberg: Springer-Verlag, 2003, pp. 1-21.

[7] D. Singh, P. Kumar, and S. Kumar, "Gap analysis between theory and practice of software testing methodologies in indian IT industry," International Journal of Computer Applications (0975-8887), vol. 101, no. 11, pp. 25-33, Sep. 2014.

[8] "European Software Testing Innovation Alliance homepage," https:// www.linkedin.com/groups/8437371, last visited: 2016-01-19.

[9] “International Software Testing Qualifications Board homepage," http: //www.istqb.org/, last visited: 2016-01-19.

[10] "Conference list by Jan Tretmans," http://www.cs.ru.nl/ tretmans/ TestConferences.html, last visited: 2016-01-19.

[11] "Conference list by Software Testing Stuff," http://www.testingstuff. com/conferences.html, last visited: 2016-01-19.

[12] B. Vasilescu, A. Serebrenik, T. Mens, M. G. van den Brand, and E. Pek, "How healthy are software engineering conferences?" Science of Computer Programming, vol. 89, Part C, pp. 251 - 272, 2014.

[13] B. Kitchenham and S. Charters, "Guidelines for performing systematic literature reviews in software engineering," Technical report, EBSE Technical Report EBSE-2007-01, Tech. Rep., 2007.

[14] "Speak Easy homepage," http://speaking-easy.com/conferences, last visited: 2016-01-19. 